\title{
Information underpinning anticipation of goal-directed throwing
}

\author{
Michael Bourne - Simon J. Bennett • Spencer J. Hayes • \\ Nicholas J. Smeeton • A. Mark Williams
}

Published online: 27 June 2013

(C) Psychonomic Society, Inc. 2013

\begin{abstract}
We identified the information used to anticipate throw direction in handball. In two experiments, we examined how anticipation performance is affected when the information from one of five body areas (right arm, shoulders, hips, trunk, or total throw side) was either neutralized or decoupled from the motion of other body segments. In the first experiment, performance was significantly reduced when information from the throwing arm was neutralized, irrespective of skill levels. Skilled participants were negatively affected when the shoulders, hips, and trunk were neutralized, whereas less-skilled participants showed trends toward improvement under identical conditions. In the second experiment, partially disrupting relative motion via decoupling was not enough to reduce the anticipation performance among skilled participants to chance levels, whereas less-skilled participants lost their ability to anticipate in three conditions. Our findings suggest that skilled and less-skilled participants employ different information extraction strategies, yet information from the throwing arm is critical to anticipation for both groups. The two experiments suggest that relative motion mediated by both the absolute displacement trajectories of individual marker locations and their relative timings are important in informing anticipation, irrespective of skill level.
\end{abstract}

M. Bourne $\cdot$ S. J. Bennett $\cdot$ S. J. Hayes

Research Institute for Sport and Exercise Sciences, Liverpool John

Moores University, Liverpool, UK

N. J. Smeeton

Chelsea School of Sport, University of Brighton, Brighton, UK

A. M. Williams

Centre for Sports Medicine and Human Performance, Brunel

University, Brunel, UK

M. Bourne $(\bowtie)$

National Cricket Performance Centre, Loughborough University, Loughborough LE11 3TU, UK

e-mail: michael.bourne@ecb.co.uk
Keywords Perception $\cdot$ Motion information $\cdot$ Skill

In recent years, methods developed for the study of biological motion perception have enhanced our understanding of how athletes anticipate an opponent's actions. Following on from the seminal work of Gunnar Johansson (1973), researchers have shown that individuals can successfully anticipate when actions are presented as point-light displays (e.g., Abernethy, Gill, Parks, \& Packer, 2001; Shim, Chow, Carlton, \& Chae, 2005; Ward, Williams, \& Bennett, 2002) or stick-figure images (e.g., Huys, Smeeton, Hodges, Beek, \& Williams, 2008; Smeeton \& Huys 2011; Williams, Huys, Cañal-Bruland, \& Hagemann, 2009). Troje (2002) concluded that when perceiving human gait, judgment is informed by the interplay between motion-mediated structural information and true, dynamic biological motion information. It is now well accepted that the "motion" component of biological motion is the more informative aspect of the stimuli (see Troje 2008).

The characteristics of the motion component that guide anticipation judgments, including the "types" of motion and their spatial location within a stimulus, are less well defined. Johansson (1973) outlined a perceptual functionality based on the perception of both common motions and the deviations of points relative to this "reference" motion. Cutting and Proffitt (1982) suggested a modified model of motion perception that involved a heuristic termed "the minimum principle," according to which motion in the optic array is minimized to its component common or relative motions. Cutting and Proffitt theorized that the initiation of the minimization process begins with both relative and common motions being minimized. One aspect is minimized before the other, and this first aspect of motion to be minimized serves as the "reference" motion, whereas the second motion component is determined as a "residual." Cutting and Proffitt suggested that in the majority of cases, including the perception of human walking, relative motion is minimized first and forms the basis of the informational value of 
biological motion (cf. Breslin, Hodges, Williams, Curran, \& Kremer, 2005; Troje in press; Williams, Ward, Smeeton, \& Allen, 2004).

To identify pertinent motion information, several researchers have used the assumption-free analysis technique principal components analysis (PCA). PCA has successfully identified the low-dimensional dynamical structures that capture pertinent motion information (e.g., Daffertshofer, Lamoth, Meijer, \& Beek, 2004; Forner-Cordero, Levin, Li, $\&$ Swinnen, 2005; Troje 2002). Furthermore, the extent to which anatomical locations contribute to a dynamical structure can also be determined from the outputs of PCA. In a PCA, these dynamical structures are represented as eigenvectors $v^{\rightarrow k}$, commonly referred to as "modes." Each mode captures a proportion of the total variance explaining the original data set, with the lowest-numbered mode (1) accounting for the largest proportion of the variance, and the highest-numbered mode $(1+n)$ accounting for the least. The total number of modes is equal to the original number of variables entered into the PCA. For comprehensive reviews of the PCA process, see Daffertshofer, Lamoth, Meijer, and Beek (2004) and Jolliffe (2002)

It has been argued that PCA can also be used to capture the informational content of motion for perceptual purposes (cf. Huys et al. 2009), since the resultant dynamical structures are directly informational (e.g., Kelso 1993). This argument has led to PCA being used to identify anatomical areas that could offer informative motion cues. Huys et al. (2008) used PCA to determine the dynamical information underpinning the perception of tennis forehand drive shots for two directions and distances (represented in PCA as modes). These authors reported that shots to different sides of the court could be predominantly, but not exclusively, characterized by how marker locations representing the right shoulder-racket linkage projected differently onto a single mode as a consequence of the shot type. These different projections were represented by significant differences in the eigenvector coefficients from the first five modes, which captured approximately $95 \%$ of the variance in the ground stroke. Huys et al. (2008) reconstructed the biological motion contained in tennis forehand shots as stick figures by using dynamics from selected PCA modes to assess anticipation behavior in skilled and less-skilled tennis players. The variance captured in the principal modes was sufficient to inform anticipation in a skill-dependent manner.

Huys and colleagues (e.g., Huys et al. 2009; Williams et al. 2009) were able to demonstrate that participants could anticipate the direction of forehand drive shots in tennis when presented with various low-dimensional representations. Furthermore, the concept of "local" or "global" information extraction grew as a topic of discussion. Researchers had previously demonstrated that observers of dot motion stimuli are able to extract the mean shared direction of motions from "local" and "global" dot arrays independently (Watanabe and Kikuchi 2006). In this context, "local" defines motion signals that are tightly grouped within a subarea of an array, whereas "global" defines the integration of motion signals spread widely across the array. Huys et al. (2008) identified a "global" spread of anatomical locations that differed significantly between tennis strokes. Following on from the argument of Kelso (1993) and Haken (2000) that the dynamics of macroscopic motion structures and visual perception information are inextricably linked, Huys et al. (2008) hypothesized that higher levels of anticipation skill would be underpinned by the "global" extraction of informative cues. Huys et al. (2009) subsequently reported that skilled anticipators in tennis employ global information extraction strategies, whereas less-skilled anticipators rely mainly on extracting information localized around the armracket linkage.

Although PCA has identified the location of dynamical information underpinning anticipation in tennis, in other, similar goal-directed sports, researchers have found that the modes capturing the greatest variance do not contain eigenvector coefficient differences corresponding to target location. Bourne, Bennett, Hayes, and Williams (2011) employed three separate PCAs to determine whether a low-dimensional representation of biological motion exists for handball penalty throwing at four separate targets. Specifically, in addition to running a PCA encompassing the motion data for all targets, as well as a separate PCA on the data for individual targets in order to characterize targetspecific dynamical structures, they sectioned the original time-series data set containing all trials to all targets into equal thirds, each representing distinct time windows in the throwing action. Although PCA did consistently identify a low-dimensional representation of the handball penalty throw, and though differences were observed at the level of the corresponding time evolution, target-specific differences at the level of the eigenvector coefficients were not identified.

With the weight of evidence suggesting that anticipation of full-body motion is underpinned by the informational content of biological motion, it is necessary to investigate further the contradictory findings reported by Bourne et al. (2011). We extended upon the research of Bourne et al. by using behavioral methods to identify whether biological motion underpins anticipation of the handball penalty throw. In the first instance, we investigated whether handball penalty throws can be anticipated using only biological motion. Furthermore, we attempted to identify the anatomical body areas that contain the information necessary for anticipation above chance levels. In recent studies, the latter aim has been addressed by manipulating the information contained within various anatomical areas by occluding and neutralizing (Huys et al. 2009), interchanging (Williams et al. 2009), 
or inverting (Blake and Shiffrar 2007; Thurman, Giese, \& Grossman, 2010; Troje, Sadr, Geyer, \& Nakayama, 2006) the information from different locations.

In this article, we applied manipulations involving neutralization and decoupling. We used two manipulations to investigate the extent to which the informational value of relative motion is underpinned by absolute location trajectories and the relative timing between these trajectories. In a neutralization condition, target-specific marker location displacement information was removed and replaced with a mean representation of marker location displacement from multiple goal-directed motions. As a consequence, the individual marker location information was removed, modifying the relative motion within the neutralized area and across the display as a whole. In a decoupling condition, the relative timing between a decoupled area and the rest of the structure was altered. By manipulating timing rather than individual location trajectories at the decoupled locations, only interarea relative motion was disrupted.

An additional aim was to determine whether any observed differences in biological motion perception could be accounted for by skill level. Notably, as we were interested in improving understanding of the information extraction strategies underpinning successful anticipation, skill classification was based solely on anticipation ability (i.e., a within-task criterion was applied so that participants were assigned to skilled or lessskilled groups on the basis of their performance when anticipating a nonmanipulated handball throw control condition), rather than on other physical or performance criteria (see Savelsbergh, Van der Kamp, Williams, \& Ward, 2005; Vaeyens, Lenoir, Williams, \& Philippaerts, 2007). Although we acknowledge that some have been concerned that a withintask criterion may reduce analytical power (e.g., Preacher, Rucker, MacCallum, \& Nicewander, 2005) or challenge the assumptions of ANOVA procedures, the prevalence of published research in the area utilizing this method leads us to conclude that the approach is justified for the present purposes.

Therefore, within our defined skill groups, the data manipulations enabled us to understand whether skill-based differences could be found in information extraction strategies.

We hypothesized, on the basis of previous research findings (i.e., Huys et al. 2009; Huys et al. 2008; Williams et al. 2009), that participants would be able to anticipate above chance levels when presented with 14-point, full-body stickfigure stimuli of handball penalty throws, irrespective of skill level. In addition, we hypothesized that the anatomical regions closest to the ball would be most informative for anticipation, and consequently, that manipulation of these areas would result in the largest decrement in performance relative to the nonmanipulated full-body stimuli across the skill levels. Specifically, we expected manipulations influencing the throwing arm to result in anticipation performance at chance levels. It was expected that because skilled participants make greater use of "global" than of "local" perceptual strategies (see Huys et al. 2009; Williams et al. 2009), the manipulation of locations away from the throwing arm would reduce the performances of the skilled participants to a greater extent than those of less-skilled participants.

\section{Experiment 1}

\section{Method}

Participants A total of 30 male athletes were recruited, with a mean age of 23.27 years ( $S D=5.56$ years). The participants had engaged in playing handball at school, university, club, or international level for a mean of 5.03 years $(S D=$ 5.25 years), and all were familiar with the throwing action. Participants gave their informed consent prior to taking part, and the experiment was conducted in accordance with the ethical guidelines of the lead institution.

Apparatus and test stimulus production The test stimuli were point-light stick figures of handball throws generated in MATLAB (MATLAB R2007b; The MathWorks) and saved in AVI format. The stimuli were constructed using three-dimensional motion data and represented individual penalty throws from five international-level, male handball players (age $=24.5$ years, $S D=2$ years; playing experience $=$ 10.6 years, $S D=4.6$ years) captured using a 14 -marker model. The players represented in the test stimuli played in attacking positions and were rated by coaching staff as the best shooters in the squad. A detailed description of the motion data capture and processing procedures are reported in Bourne et al. (2011).

All neutralization and decoupling manipulations were applied to the time-series data from the original stimulus clips. These procedures were expected to result in minor modifications of the relative distances between markers at certain points in the motion, relative to the nonmanipulated conditions. Perceptual judgments based on biological motion have proven to be robust under other instances of modified structure. For example, recognition of human motion has been shown to be effective when the points in the display are selectively presented or occluded over time (Neri, Morrone, \& Burr, 1998). Moreover, observers are able to extract displacement direction from a display even when the display is scrambled (Troje and Westhoff 2006) or spatially degraded (Casile and Giese 2005). The motion component of biological motion is thought to be the information source underpinning action recognition (see Blake and Shiffrar 2007; Troje 2008). Additionally, because we used stick figures rather than point-light display stimuli in the present experiments, participants were not required to identify structure-from-motion, as the structure of human 
geometry was readily identified through the explicitly drawn point linkages (see Troje 2002). The structure of a human actor was available throughout all trials, even though the distortion of the structure may have appeared somewhat "unnatural" in some decoupling conditions. The findings of previous research and the use of explicitly defined stick figures led us to believe that transient minor structural changes to the display were unlikely to impact on anticipation performance or to confound the effect of changes to relative motion. Fig. 1 provides an example of a decoupled stimulus at the point where the peak delay threshold has been reached, as compared with its nonmanipulated counterpart.

The test stimuli in Experiment 1 represented either an original penalty shot or a penalty shot with some points in the display neutralized. Neutralized points represented an average time-series displacement for that location, for that shooter only, across shots to all four of the targets (i.e., top left [TL], top right [TR], bottom left [BL], and bottom right $[\mathrm{BR}])$. The locations of targets were defined relative to the viewing position of the participant (see Fig. 2). Therefore, the data in a neutralized stimulus clip were a mix of a single shot, for the nonneutralized points, and a representative average of multiple shots to the four targets from a single shooter, for the neutralized points. Six conditions were represented in stick-figure format: control (14-point/14-segment stick figure minus ball), arm neutralized (r-shoulder, $\mathrm{r}$ elbow, r-wrist), shoulders neutralized (r-shoulder, 1shoulder), hips neutralized (r-hip, 1-hip), trunk neutralized (r-shoulder, 1-shoulder, r-hip, 1-hip), and total throw side neutralized (r-shoulder, r-elbow, r-wrist, r-hip, r-knee, rankle, r-foot). Figure 3 outlines the locations manipulated under each condition. Twenty clips were created per

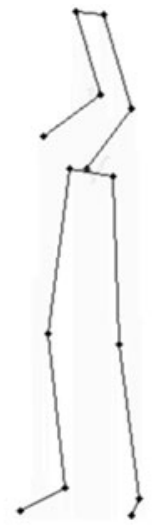

Control Stimuli

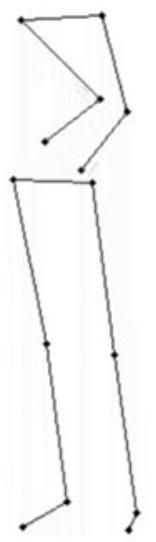

Equivalent Trunk Decoupled Stimuli

Fig. 1 Visual representation of the difference between a decoupled stimulus and its control equivalent at the point in time at which maximum decoupling is reached. The presented example represents the trunk decoupled condition
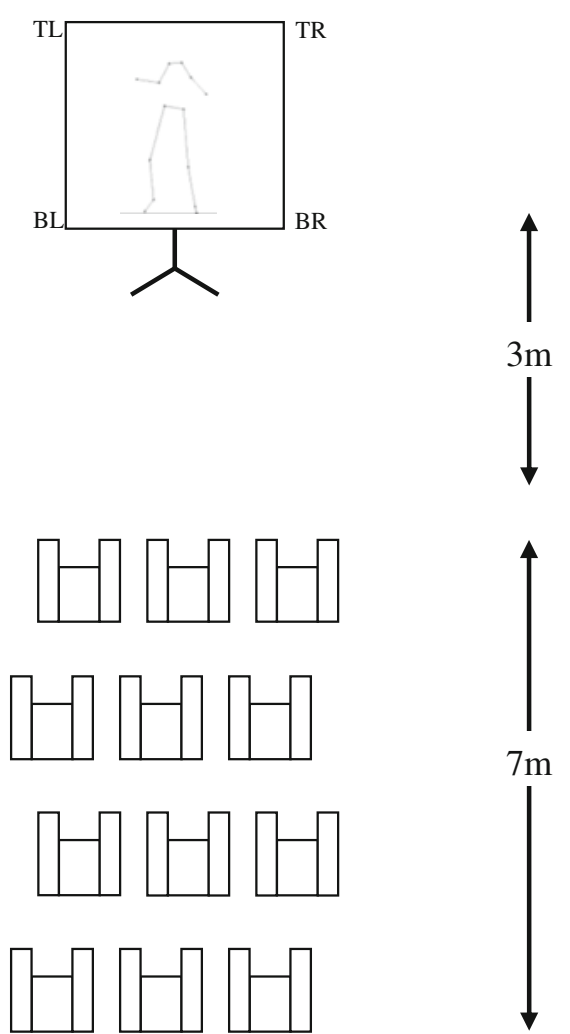

Fig. 2 Overhead diagram representing the layout of the data collection setup

condition and were combined within MATLAB to generate two test films consisting of 60 trials each $(N=120)$. The trials were randomly sequenced within and across the test films, such that each contained an equal number of stimulus clips for each condition. The intertrial interval was $3 \mathrm{~s}$, and each clip lasted for $2 \mathrm{~s}$. A practice film involving 12 trials was constructed using the same procedure as the test film.

Procedure Participants were tested as a group. The practice and test films were presented on a $3 \mathrm{~m}(\mathrm{~h}) \times 2 \mathrm{~m}(\mathrm{w})$ projection screen from a Sony VPL-EW130 3000 ANSI Lumens $1,280 \times 800$ projector (Sony Inc, Tokyo Japan). Participants were seated from 3 to $10 \mathrm{~m}$ from the screen, such that the image subtended average visual angles of $35^{\circ}$ in the vertical and $24^{\circ}$ in the horizontal axis. Pilot testing indicated that seating position was not associated with response accuracy. Participants were informed that they would be shown handball penalty throws to one of four different targets. They were asked to imagine that they were between the thrower and the goal and to anticipate which target area the ball would be thrown towards. Participants were informed that the stimuli would involve right-handed stick figures constructed from black markers representing 14 major joints of the body, but with no ball or ball flight information. 


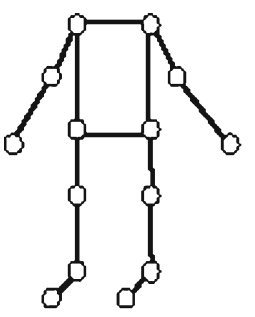

CONTROL

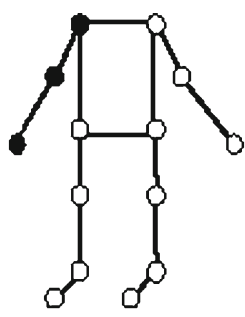

ARM

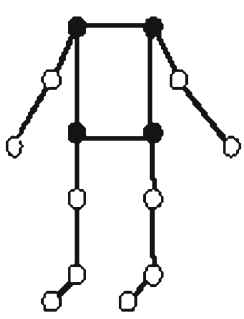

TRUNK

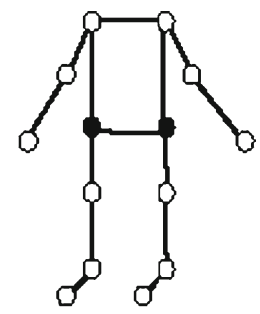

HIPS

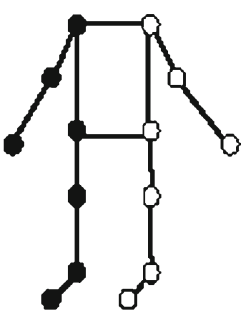

TOTAL THROW SIDE (TTS)
Fig. 3 Visual representation of the marker locations that were (a) neutralized or (b) decoupled in each condition of Experiments 1 and 2 , respectively

Participants were instructed to make their anticipation judgments immediately after the clip had been shown, using pen and paper. After this initial instruction, the group responded to 12 trials of known target locations, in order to allow for familiarization with the test conditions. A short break was given before presentation of the test films, during which time participants were free to ask questions. The two 60 -trial test films were presented with a break of $4 \mathrm{~min}$ between, with each session lasting approximately $25 \mathrm{~min}$.

Data analysis The number (c) of correct answers was calculated for target accuracy. Each participant was classified as being high, medium, or low performing on the basis of response accuracy in the control condition, using a withintask criterion. Participants were ranked on a scale from 1 to 30 , after which a tertile split was applied to give the following perceptual skill groups: $1-10=$ skilled, $11-20=$ intermediate, 21-30=less skilled. As a number of participants were equally ranked on the control condition, the groups contained unequal numbers of participants $(12,8$, and 10 , respectively). The data from the intermediate group were removed from the analysis.

All of the response data $(c)$ were transformed using Bartlett's modified arcsine transformation, $p^{\prime}=(360 / 2 \pi)$ $\arcsin (\sqrt{(c+3 / 8) /(n+3 / 4)})$, where $n$ represents the number of trials in the condition. The resulting $p^{\prime}$ values were entered into an analysis of variance (ANOVA) with condition (6) as the repeated measure and Perceptual Skill
Level as the fixed factor. Where sphericity was violated, degrees of freedom were adjusted using the Huynh-Feldt correction. Fisher's least significant difference post-hoc tests were used to investigate the location of any significant differences. All means, standard deviations, and percentages reported are representative of the original response data. To examine whether any of the neutralization conditions resulted in response performances at chance levels, we compared the performances in each condition to the $25 \%$ guessing criterion using single-sample $t$ tests.

Results

Effect of neutralization condition on response accuracy We found significant main effects for skill level $[F(1,20)=$ $12.08, p<.01]$ and for condition $[F(3,58)=49.36, p<.001]$. These main effects were superseded by a significant Skill Level $\times$ Condition interaction $[F(3,58)=8.62, p<.001]$. The skilled group's response accuracy was significantly higher $(p<.01)$ than that of the less-skilled group in the control (64 \% vs. $36.5 \%$ ) and trunk (59.5\% vs. $43.5 \%$ ) conditions. The skilled group exhibited a significant decrease in response accuracy $(p<.01)$ under the arm $(M=23 \%, S D=$ $6.5 \%)$, hip $(M=54.5 \%, S D=11 \%)$, shoulder $(M=53.3 \%$, $S D=9 \%)$, and total throw side $(M=19 \%, S D=6 \%)$ conditions relative to control $(M=64 \%, S D=4.5 \%)$. For the lessskilled group, the $\operatorname{arm}(M=28 \%, S D=12 \%)$ and total throw side $(M=22 \%, S D=7.5 \%)$ conditions resulted in significantly decreased response accuracy $(p<.05)$ as compared to the control condition $(M=36.5 \%, S D=10 \%)$, whereas the shoulder condition $(M=47.5 \%, S D=16.5 \%)$ resulted in significantly increased anticipation performance as compared to control $(p<.05)$.

Response accuracy relative to chance Participants anticipated target locations at levels significantly above chance in the control, hip, shoulder, and trunk conditions, irrespective of skill level. The arm and total throw side manipulations produced accuracy at chance levels for both skill groups. The results are presented in Fig. 4.

\section{Discussion}

We examined whether skilled and less-skilled participants are able to anticipate throw direction on the basis of the information presented in stick-figure stimuli across six conditions: control, arm neutralized, hips neutralized, shoulders neutralized, trunk neutralized, and total throw side neutralized. We predicted that participants would anticipate above chance level when presented with stimuli that were not manipulated (control), but that performance would be 
Fig. 4 Bar graph presenting the mean percentage response accuracy of skilled and lessskilled participants under control and the five neutralized manipulation conditions. Error bars represent the response accuracy standard deviations for the condition. Asterisks $(*)$ denote that the response accuracy of the skilled participants was significantly better than that of their lessskilled counterparts, and delta $(\Delta)$ symbols denote significant differences between the manipulation condition and the control condition

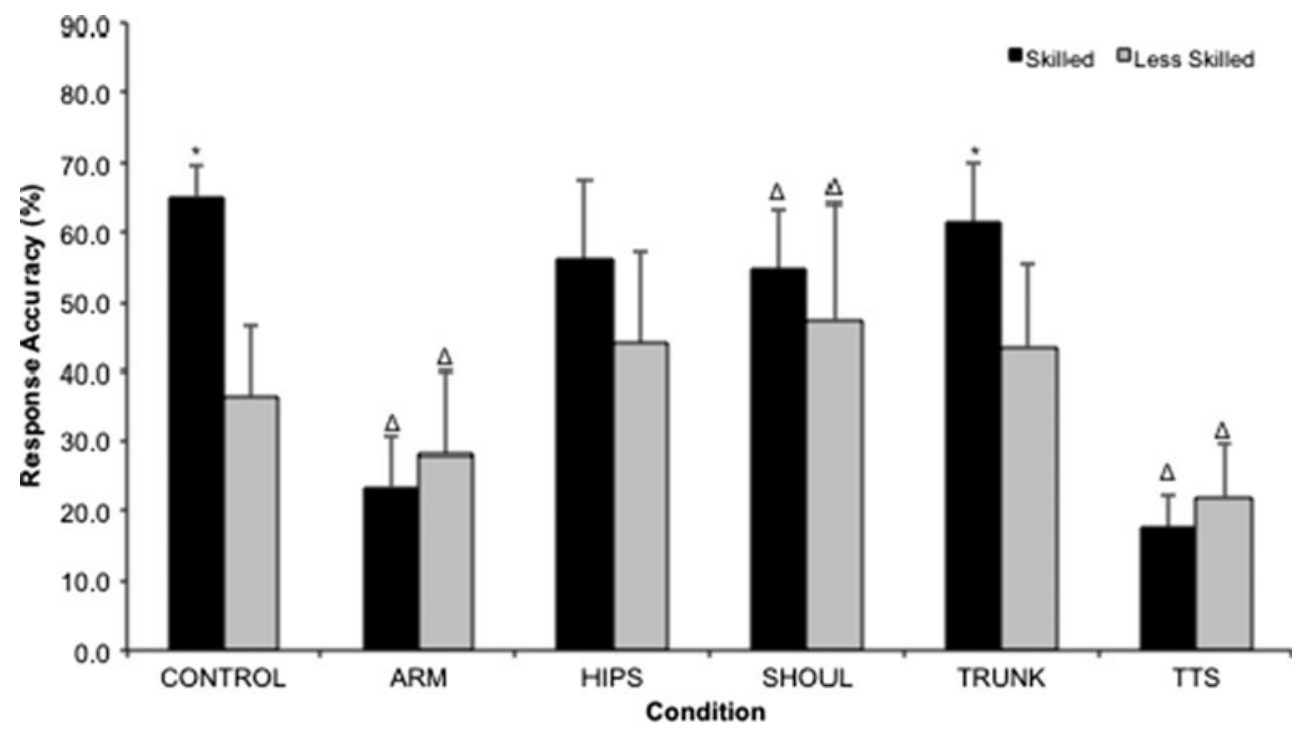

reduced to chance levels when the arm was neutralized, due to the removal of target-specific information from the three marker locations most proximal to the ball. We predicted that the neutralization of locations away from the arm would reduce the ability of the skilled participants to a greater extent than that of the less-skilled individuals, as skilled participants are thought to make greater use of more global sources of motion information.

Participants were able to anticipate shot direction above chance levels when presented with nonneutralized stimuli (control), confirming that the information contained in the 14-point stick-figure representations contained sufficient information to facilitate anticipation. This finding is in agreement with previous reports involving goal-directed actions (cf. Huys et al. 2009; Huys et al. 2008; Shim, Carlton, \& Kwon, 2006). A comparison of the present findings to those of Bourne, Bennett, Hayes, and Williams (2011) suggests that other information beyond the low-dimensional representation of the handball penalty throw captured through the PCA process is required in order to enable accurate anticipation of the handball throw.

Bourne et al. (2011) required four modes to capture $>90 \%$ of the variance explained by PCA. However, unlike the Huys et al. (2008) analysis of the tennis forehand drive, the information captured in the modes identified by Bourne et al. was insufficient to differentiate statistically between the throws to different locations at the level of the eigenvector coefficients. Bourne et al. did note differences between shots at the level of the corresponding time evolutions, which may reflect the findings in the present article. Furthermore, the processing or analysis procedures used by Bourne et al. may have removed some information that was helpful in the present case. As one example, motion amplitudes were normalized in the original data sets, as had been suggested by Daffertshofer et al. (2004) to guard against the PCA being biased toward marker locations with the largest displacement amplitudes. If amplitude information is utilized in the anticipation process (see Smeeton and Williams 2012), the PCA applied by Bourne et al. could have impacted on performance.

Skill-based differences in information extraction The skilled perceptual group outperformed the less-skilled perceptual group under all conditions that did not neutralize the arm, although not significantly so in all cases. Furthermore, neutralization of the arm resulted in accuracy at chance levels, irrespective of skill level. These findings are consistent with those of Huys et al. (2008) and Williams et al. (2009), who reported that information contained in areas "local" to the ball (i.e., the end effector) are most useful for anticipating target location. Moreover, research involving handball throwing has often identified the mechanics of the arm as being a key factor in ball flight trajectory (Fradet et al. 2004; Wagner, Klous, \& Muller, 2006). It appears that neutralization of the target-specific information within the wrist, elbow, and shoulder left participants to rely on less pertinent sources of information, such as the hips and left shoulder.

The nature of the skill-based difference is best explained under shoulder-neutralized conditions. The performance of the skilled group was significantly reduced when the shoulders were neutralized, whereas the performance of the lessskilled group was significantly improved under the same conditions. These findings indicate that the ability to extract information from these locations varies between skill groups. The skilled group's ability to extract information from a wider range of locations away from the end effector, as compared to less-skilled participants, has been explained previously in the context of the "global" extraction of information (Huys et al. 2009; Williams et al. 2009). Under conditions in which goalspecific shoulder motion was present, less-skilled participants may have been deceived (see Jackson, Warren, \& Abernethy, 
2006) or confused by motion that they were sensitive to but not able to interpret reliably.

The present experiment did produce some potentially confounding findings which are worthy of further exploration. Neutralization of the shoulders modifies information within the throwing arm (right shoulder), which was shown here to be critical to successful anticipation for all skill groups. Thus, the removal of target-specific motion from the right shoulder was associated with significant increases and decreases in the performance of less-skilled participants, depending on the structure neutralized. Observational learning studies have suggested that the information extracted from the end effector of an action is sufficient to facilitate skill learning (Hodges, Hayes, Breslin, \& Williams, 2005), and this may offer some insight into the processes employed during anticipation. It may thus be that in the case of less-skilled participants, "localized" refers mostly to single end-effector locations rather than whole limbs. Under these circumstances, motion information from the shoulders may confound less-skilled participants, and its removal thus makes anticipation judgments more straightforward. The methods required to investigate this theory will be explored further in the General Discussion.

\section{Experiment 2}

In Experiment 1, we reported that neutralizing the targetspecific information contained in the throwing arm, shoulders, and to a lesser extent the hips induced significant changes in anticipation performance in both skilled and less-skilled perceivers. In the case of neutralizing information in the shoulders, the direction of the effect differed between the skill groups. The neutralization process removes target-specific displacement of marker locations, which has a knock-on effect on intra- and inter-body-area relative motion. Whether the negative impact on anticipation from neutralizing a body area such as the arm was due to the loss of information from the target-specific displacement trajectories of the marker locations only, or rather to the disruption of relative timing, could not be determined. In Experiment 2, we examined the effect on anticipation of disrupting only the inter-body-area relative timing. The disruption of relative timing was achieved by decoupling the timing of displacement between specific areas of the display (e.g., between right arm locations and the rest of the display). The procedure of decoupling holds the individual marker-location displacement trajectories intact, which allows for any effects in response accuracy to be isolated to the information resulting from relative timing.

We predicted that decoupling the throwing arm, shoulders, trunk, and the total throw side independently would significantly reduce performance relative to the control condition for skilled participants, due to the disruption around the shoulder region. Performance of the less-skilled participants was predicted to be at chance levels under the shoulder and trunk decoupled conditions, as these disrupt relative motion within the throwing arm. We also hypothesized that skilled participants would retain their superiority over lessskilled counterparts under all conditions, due to the targetspecific displacement information from all marker locations being available for extraction globally. Finally, we expected performance to be maintained above chance levels for skilled participants, because a higher level of targetspecific motion information was retained than in the previous neutralized conditions.

\section{Method}

Participants The participants were male athletes $(N=19)$ with a mean age of 24.24 years $(S D=6.33$ years). They had engaged in playing handball at school, university, club, or international level for an average of 5.24 years $(S D=$ 5.57 years). The participants gave informed consent prior to taking part, and the experiment was conducted in accordance with the ethical guidelines of the lead institution. None of the participants had taken part in Experiment 1.

Apparatus and test film production Each of the stimuli contained data from a single shot. To create decoupled locations within the stimulus clips, time-series data were manipulated such that the initiation of displacement for these decoupled locations was delayed by $25 \%$ of the original movement time. To retain the structure of the display, the first data point was displayed during the delay period for the decoupled locations, and the last data point of the nondecoupled locations was displayed until the completion of the clip. The aim of the decoupling approach was to disrupt the interlocation relative motion with respect to the control condition, and thereby to determine the effect of this motion on anticipation performance. The choice of a $25 \%$ decoupling over a larger or smaller percentage was based on pilot work, which led us to expect that this would be sufficient to modify the information available for anticipation.

Six conditions were presented in Experiment 2: control (14-point/14-segment stick figure minus ball), right arm decoupled (r-shoulder, r-elbow, r-wrist), shoulders decoupled (1-shoulder, r-shoulder), hips decoupled (1-hip, r-hip), trunk decoupled (1-shoulder, r-shoulder, 1-hip, rhip), and total throw side decoupled (r-shoulder, r-elbow, r-wrist, r-hip, r-knee, r-ankle, r-foot).

Procedure and data analysis The procedure and data analysis methods were similar to those in Experiment 1. As a result of the decoupling method, the clips lasted for 3 rather than 2 s. Also, due to a smaller sample size, a mid-group split was applied, so that those ranked 1-10 were classified 
as the perceptually skilled group and those ranked $11-19$ as the less perceptually skilled group.

Results

Effect of decoupling condition on response accuracy $\mathrm{A}$ significant main effect was reported for skill level $[F(1$, $17)=23.29, p<.001]$, which was superseded by a significant Skill Level $\times$ Condition interaction $[F(5,85)=3.88, p<.001]$ (see Fig. 5). The skilled group's response accuracy scores were significantly higher $(p<.05)$ than those of the lessskilled group in the control (56 \% vs. $29.5 \%$ ) and shoulder decoupled (59\% vs. $31.5 \%$ ) conditions. The skilled group recorded significantly lower accuracy scores $(p<.05)$ under the total throw side decoupled $(M=47.5 \%, S D=14 \%)$ condition relative to the control condition $(M=56 \%, S D=$ $6 \%)$. For the less-skilled group, the hips decoupled ( $M=$ $43.5 \%, S D=13.5 \%)$ and total throw side decoupled $(M=$ $40 \%, S D=15.5 \%$ ) conditions resulted in a significant increase in response accuracy as compared to the control condition $(M=29.5 \%, S D=10 \%)$.

Response accuracy relative to chance The skilled group was able to anticipate above chance levels under all conditions, whereas the less-skilled group was only able to anticipate significantly above chance under the control, hips, and total throw side conditions.

\section{Discussion}

We examined the effect of decoupling the relative timings of marker location displacement on the anticipation of shot direction across skilled and less-skilled participants.
Participants were asked to anticipate throw direction under six conditions: control, arm decoupled, hips decoupled, shoulders decoupled, trunk decoupled, and total throw side decoupled. We hypothesized that skilled participants would show reduced accuracy scores under the arm, shoulder, trunk, and total throw side conditions, due to decoupling disrupting the relative motion information around the shoulder region. Additionally, we hypothesized that the lessskilled group's anticipation performance would be significantly impaired in the shoulder and trunk conditions, which decouple locations within the throwing arm. Furthermore, we predicted that the skilled participants would maintain their perceptual superiority across all conditions.

Our findings corroborate those presented in Experiment 1. The information contained in the nonmanipulated (control condition) stick-figure stimuli was enough for participants to consistently anticipate above chance levels. Stick-figure motion of handball throwing is thus taken to contain the minimum information necessary to facilitate anticipation judgments. As hypothesized, none of our decoupling manipulations resulted in anticipation being reduced to chance levels in the perceptually skilled group. Altering the relative motion between selected body areas by using a decoupling manipulation did not appear to remove the minimum information necessary to distinguish shot direction for the skilled anticipators. Overall, decoupling appeared to be less disruptive to skilled anticipators than was the equivalent neutralization condition. For instance, where the anticipation ability of skilled participants was lost under the arm and total throw side neutralized conditions, the ability was retained under decoupling, even though this ability was significantly reduced relative to control when the total throw side was decoupled. These findings suggest that intralimb relative motion may be of informational value to skilled anticipators.
Fig. 5 Bar graph presenting the mean percentage response accuracy of skilled and lessskilled participants under control and the five decoupled conditions. Error bars represent the response accuracy standard deviations for the condition. Asterisks $(*)$ denote that the response accuracy of the skilled participants was significantly better than that of their lessskilled counterparts, and delta $(\Delta)$ symbols denote significant differences between the manipulation condition and the control condition

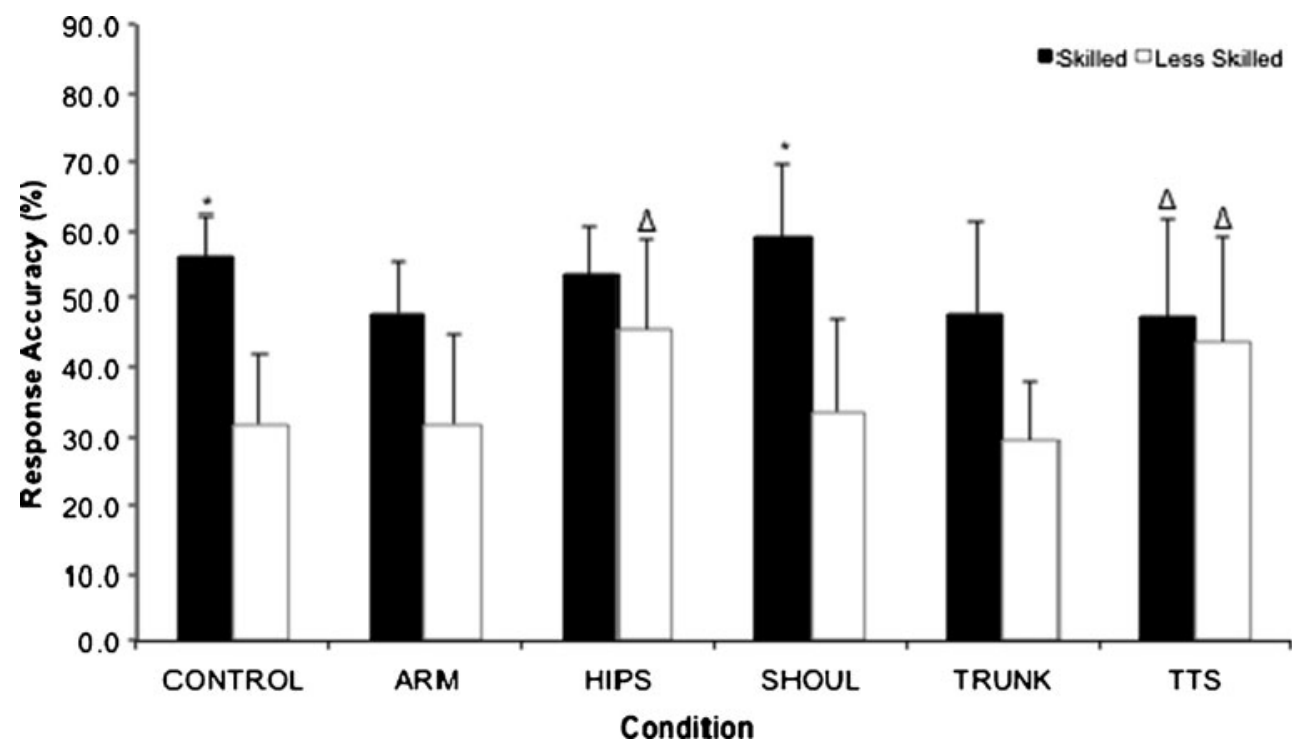


The performance of the less-skilled group fell to chance levels when the arm, trunk, and shoulders were decoupled, demonstrating that unlike in the skilled participants, the disruption of relative timing was enough to impair the anticipation ability of less-skilled participants in some instances, even when the localized arm information was intact. It should be noted, though, that under these circumstances, performance was not significantly below that in the control condition, due to the relatively low anticipation abilities of the less-skilled group overall. Thus, it took only minor degradations in performance under decoupled conditions to reduce anticipation to chance levels, which was not the case among the skilled participants. A further unexpected occurrence, however, was the significant increase in the anticipation performance of less-skilled participants when the hips and total throw side were decoupled. These findings suggest a sensitivity to "globally" distributed motion in lessskilled participants, a characteristic also identified under shoulder neutralization in Experiment 1. The anticipation performance of the less-skilled participants may still be based principally on successfully extracting information from around the throwing arm (see also Huys et al. 2009; Huys et al. 2008), but the present findings suggest that the less-skilled participants were also sensitive to motion beyond the "local" regions. It would appear that global sensitivity was not paired with global information extraction in those less-skilled participants, however, and this may have resulted in some global motion proving confounding for these participants. In this regard, we take "sensitivity" to refer to perceptual stimulation from motion, whereas "extraction" relates to the ability to draw anticipation-specific meaning from the motion. The need to separate sensitivity to and successful extraction of information into different entities is supported by Smeeton and Huys (2011), who referred to the ability to separate informative relative motion from "noise" as being crucial when anticipating tennis forehand shots. In the present case, it would be necessary to quantify what motion characteristics are perceived as being "noise" by less-skilled participants before a full explanation of the observed performance increases under motion-degraded conditions could be provided.

In sum, we conclude that the interarea relative timing of motion is informational in a skill-dependent manner, with its disruption having a variable impact on less-skilled participants, whereas skilled participants are only negatively affected.

\section{General discussion}

We showed that skilled and less-skilled participants are able to anticipate throw direction above chance levels using the information contained in full stick-figure representations of handball penalty shots. We suggest, therefore, that the minimum information necessary to anticipate target-specific differences in handball penalty throws is captured in biological motion. We also suggest that PCA can be a useful tool for identifying differences in dynamical structure, albeit researchers should be open-minded regarding alternative approaches to PCA application and interpretation.

The findings reported in Experiment 1 suggest that information from the right arm strongly influenced anticipation judgments, irrespective of skill level. The importance of the right arm locations was reflected in both the arm and total throw side conditions. In Experiment 2, we offered some support for the importance of the throwing arm. We suggest that the importance of the right arm is a consequence of its proximity to the to-be-thrown ball, and hence the use of this information does not seem to distinguish between skill levels. Whether the throwing arm is truly informational in its own right, or only as part of more information-rich fullbody stimuli, should be determined in future study. In the present article, we have shown that the lines between local and global perception can be blurred by what appear to be disconnected mechanisms for motion sensitivity and the extraction of motion information in less-skilled participants. By quantifying the informational value of throwing arm locations only, one would be able to discuss how useful true "local" information extraction strategies could be when anticipating throwing and similar fast ball tasks.

The extraction of pertinent information-from-motion other than that from the throwing arm appeared to be discriminating, with skilled participants responding negatively when target-specific information from the shoulders and hips was neutralized. Furthermore, less-skilled participants were often shown to increase their performance when nonthrowing-arm motion was neutralized or decoupled. We postulate, on the basis of our present findings and those of similar research (Huys et al. 2009; Huys et al. 2008; Williams et al. 2009), that successfully extracting targetspecific information from locations distal from the ball requires a higher level of task-specific knowledge than does drawing similar information from the proximal arm locations in fast ball sports such as handball and tennis. It appears that skilled participants are able to extract useful information from areas within a display, whereas less-skilled participants are sensitive to the specific motion but are somehow inhibited by its presence. The mechanism behind the ability to extract information globally is not clear at present, and further research will be necessary. Yet it seems clear that a global extraction strategy may allow for greater perceptual flexibility when certain information sources are constrained (cf. Smeeton and Huys 2011).

Our findings suggest that target-specific intra- and interarea relative motion couplings are informative for skilled anticipation. Single-sample $t$ test analysis showed that for skilled participants, the neutralization of target- 
specific information significantly reduced anticipation performance relative to control more than did decoupling under the arm, hips, and total throw side conditions, but that decoupling still resulted in some negative effects. Whereas neutralization removed target-specific motion information in the form of marker location trajectories, which had a knock-on effect on intra- and interarea relative motion, decoupling manipulated interarea relative timing only. An important question for researchers relates to the ratio of useful information that is extracted from relative motion between locally paired markers versus information specifically from the trajectory of individual marker locations. Cutting and Proffitt (1982) suggested that absolute motion is rarely perceived; however, this claim should be examined with a particular focus on the end effector if we are to completely model the informational properties of the goal-directed throw.

In conclusion, the biological motion of handball penalty throwing appears to provide the minimum information necessary to inform anticipation judgments. Informative motion appears to be spread "globally" across the action and to be encoded in motion at multiple levels. The manner in which this information is extracted appears to be skill dependent, and as we attempt to quantify the information most pertinent (or disruptive) to performers across the skill spectrum when making anticipation judgments, it will be helpful to investigate the informational value of individual location trajectories as well as the relative motion within specific anatomical structures.

\section{References}

Abernethy, B., Gill, D. P., Parks, S. L., \& Packer, S. T. (2001). Expertise and the perception of kinematic and situational probability information. Perception, 30, 233-252.

Blake, R., \& Shiffrar, M. (2007). Perception of human motion. Annual Review of Psychology, 58, 47-73.

Bourne, M., Bennett, S. J., Hayes, S. J., \& Williams, A. M. (2011). The dynamical structure of handball penalty shots as a function of target location. Human Movement Science, 30, 40-55.

Breslin, G., Hodges, N. J., Williams, A. M., Curran, W., \& Kremer, J. (2005). Modelling relative motion to facilitate intra-limb coordination. Human Movement Science, 24, 446-463.

Casile, A., \& Giese, M. A. (2005). Critical features for the recognition of biological motion. Journal of Vision, 5, 348-360.

Cutting, J. E., \& Proffitt, D. R. (1982). The minimum principle and the perception of absolute, common, and relative motions. Cognitive Psychology, 14, 211-246.

Daffertshofer, A., Lamoth, C. J., Meijer, O. G., \& Beek, P. J. (2004). PCA in studying coordination and variability: A tutorial. Clinical Biomechanics, 19, 415-428.

Forner-Cordero, A., Levin, O., Li, Y., \& Swinnen, S. P. (2005). Principal component analysis of complex multijoint coordinative movements. Biological Cybernetics, 93, 63-78.

Fradet, L., Botcazou, M., Durocher, C., Cretual, A., Multon, F., Prioux, J., \& Delamarche, P. (2004). Do handball throws always exhibit a proximal-to-distal segmental sequence? Journal of Sports Sciences, 22, 439-447.
Haken, H. (2000). Information and self-organization: A macroscopic approach to complex systems ( $2 \mathrm{nd}$ ed.). Berlin, Germany: Springer.

Hodges, N. J., Hayes, S. J., Breslin, G., \& Williams, A. M. (2005). An evaluation of the minimal constraining information during observation for movement reproduction. Acta Psychologica, 119, 264282. doi:10.1016/j.actpsy.2005.02.002

Huys, R., Cañal-Bruland, R., Hagemann, N., Beek, P. J., Smeeton, N. J., \& Williams, A. M. (2009). Global information pickup underpins anticipation of tennis shot direction. Journal of Motor Behavior, 41, 158-170.

Huys, R., Smeeton, N. J., Hodges, N. J., Beek, P. J., \& Williams, A. M. (2008). On the dynamic information underlying visual anticipation skill. Perception \& Psychophysics, 70, 1217-1234. doi:10.3758/PP.70.7.1217

Jackson, R. C., Warren, S., \& Abernethy, B. (2006). Anticipation skill and susceptibility to deceptive movement. Acta Psychologica, 123, 355-371.

Johansson, G. (1973). Visual perception of biological motion and a model for its analysis. Perception \& Psychophysics, 14, 201-211.

Jolliffe, L. T. (2002). Choosing a subset of principal components or variables. In L. T. Jolliffe (Ed.), Principal component analysis (2nd ed., pp. 111-149). New York, NY: Springer.

Kelso, J. A. S. (1993). The informational character of self-organized coordination dynamics. Human Movement Science, 13, 393-413.

Neri, P., Morrone, M. C., \& Burr, D. C. (1998). Seeing biological motion. Nature, 395, 894-896.

Preacher, K. J., Rucker, D. D., MacCallum, R. C., \& Nicewander, W. A. (2005). Use of the extreme groups approach: A critical reexamination and new recommendations. Psychological Methods, 10, 178-192. doi:10.1037/1082-989X.10.2.178

Savelsbergh, G. J. P., Van der Kamp, J., Williams, A. M., \& Ward, P. (2005). Anticipation and visual search behaviour in expert soccer goalkeepers. Ergonomics, 48, 1686-1697. doi:10.1080/ 00140130500101346

Shim, J., Carlton, G. L., \& Kwon, Y. H. (2006). Perception of kinematic characteristics of tennis strokes for anticipating stroke type and direction. Research Quarterly for Exercise and Sport, 77, 326-339.

Shim, J., Chow, J. W., Carlton, L. G., \& Chae, W.-K. (2005). The use of anticipatory visual cues by highly skilled tennis players. Journal of Motor Behaviour, 37, 164-175.

Smeeton, N. J., \& Huys, R. (2011). Anticipation of tennis-shot direction from whole-body movement: The role of movement amplitude and dynamics. Human Movement Science, 30, 957-965. doi:10.1016/j.humov.2010.07.012

Smeeton, N. J., \& Williams, A. M. (2012). The role of movement exaggeration in the anticipation of deceptive soccer penalty kicks. British Journal of Psychology, 103, 539-555. doi:10.1111/j.20448295.2011.02092.x

Thurman, S. M., Giese, M. A., \& Grossman, E. D. (2010). Perceptual and computational analysis of critical features for biological motion. Journal of Vision, 10(12):15, 1-14. doi: $10.1167 / 10.12 .15$

Troje, N. F. (2002). Decomposing biological motion: A framework for analysis and synthesis of human gait patterns. Journal of Vision, 2(5):2, 371-387. doi:10.1167/2.5.2

Troje, N. F. (2008). Retrieving information from human movement patterns. In T. F. Shipley \& J. M. Zacks (Eds.), Understanding events: from perception to action (pp. 308-334). Oxford, UK: Oxford University Press.

Troje, N. F. (in press). In M. D. Rutherford \& V. A. Kuhlmeier (Eds.), Social perception: Detection and interpretation of animacy, agen$c y$, and intention. Cambridge, MA: MIT Press.

Troje, N. F., Sadr, J., Geyer, H., \& Nakayama, K. (2006). Adaptation aftereffects in the perception of gender from biological motion. Journal of Vision, 6(8):7, 850-857. doi:10.1167/6.8.7 
Troje, N. F., \& Westhoff, C. (2006). The inversion effect in biological motion perception: Evidence for a "life detector"? Current Biology, 16, 821-824.

Vaeyens, R., Lenoir, M., Williams, A. M., \& Philippaerts, R. M. (2007). Mechanisms underpinning successful decision making in skilled youth soccer players: An analysis of visual search behaviours. Journal of Motor Behavior, 39, 395-408.

Wagner, H., Klous, M., \& Müller, E. (2006). Kinematics of the upward jumping throw in handball - Comparison of players with different level of performance. In H. Schwameder, G. Strutzenberger, V. Fastenbauer, S. Lindinger, \& E. Müller (Eds.), Proceedings of the 24th International Symposium on Biomechanics in Sports
[Online]. Konstanz, Germany: International Society of Biomechanics in Sports. Retrieved from https://ojs.ub.unikonstanz.de/cpa/article/view/340/296

Ward, P., Williams, A. M., \& Bennett, S. J. (2002). Visual search and biological motion perception in tennis. Research Quarterly for Exercise and Sport, 73, 107-112.

Watanabe, O., \& Kikuchi, M. (2006). Hierarchical integration of individual motions in locally paired-dot stimuli. Vision Research, 46, 82-90.

Williams, A. M., Huys, R., Cañal-Bruland, R., \& Hagemann, N. (2009). Dynamical information underpinning anticipation skill. Human Movement Science, 28, 362-370. 